\title{
Caracterización de la hemoglobina glucosilada A1c en embarazas sanas
}

\section{Characterization of glycosylatedhemoglobin Alc in healthypregnancies}

\author{
Yamilé Arencibia Fundora ${ }^{1}$, Ignacio Piloto Rivera ${ }^{2}$, Arcelia Gonzáles Paneque ${ }^{3} \&$ Merien \\ Bello Ferro ${ }^{4}$
}

\section{Recibido: 14-01-2018 / Revisado: 15-02-2018 / Aceptado: 16-03-2018 / Publicado: 05-04-2018}

\section{DOI: https://doi.org/10.33262/anatomiadigital.v1i1.1040}

\begin{abstract}
.
Introduction: in pregnant women with pregestational diabetes mellitus (type 1 or 2 ) and gestational diabetes the determination of glycosylatedhemoglobinisparticularlyimport antbecauseitscorrelationalvalue has been seen with the development of maternal and fetal complications. Objective: to characterize the glycosylated hemoglobin in the different trimesters of pregnancy.
\end{abstract}

\section{Resumen.}

Introducción: en embarazadas con diabetes mellitus pregestacional (tipo 1 o 2 2) y gestacional la determinación de la hemoglobina glucosilada tiene particular importancia pues se ha visto su valor correlacional con el desarrollo de complicaciones maternas y fetales.

Objetivo: caracterizar la hemoglobina glucosilada en los diferentes trimestres del embarazo.

\footnotetext{
1 Especialista de I grado en Medicina General Integral y en Laboratorio Clínico. Asistente. Hospital Ginecobstétrico «Manuel Fajardo». Güines, Cuba. Correo electrónico: ignaciopr@infomed.sld.cu https://orcid.org/0000-0002-7353-0188

${ }^{2}$ Especialista de I grado en Medicina General Integral. Máster en Atención Integral a la Mujer. Instructor.

Policlínico «Luis Li Trigent». Güines, Cuba. Correo electrónico: ignaciopr@infomed.sld.cu https://orcid.org/0000-0002-7353-0189

${ }^{3}$ Licenciada de Laboratorio Clínico y en Medicina Transfusional. Asistente. Hospital Ginecobstétrico «Manuel
} Fajardo». Güines, Cuba. Correo electrónico: arceliagp@infomed.sld.cu iD https://orcid.org/0000-00027353-0190

4 Especialista de I grado en Fisiología Normal y Patológica. Profesor Auxiliar. Máster en Medicina Bioenergética y Natural en la Atención Primaria de Salud y en Educación Médica. Facultad de Ciencias Médicas Mayabeque. Güines, Cuba. Correo electrónico: mbello@infomed.sld.cu iD https://orcid.org/00000002-7353-0191 
Methods: cross-sectional analytical observational study in the Manuel Fajardo Gyneco-Obstetric Hospital during he second semester of 2016 to 100 healthy pregnancies that attended the prenatal control; and a control group of 150 healthy non-pregnant women older than 17 years in the population corresponding to the municipality of Güines whomt the inclusion and exclusion criteria. Determinations of glycosylated hemoglobin and fasting glycemia were made.

Results: the healthy pregnant women the average glycosylated hemoglobin was lower than in non-pregnant women, respectively $4.50 \%(4.41-4.49)$ versus $5.21 \%$ (5.165.26), $\mathrm{P}<0.001$. In the healthy pregnant group, glycosylated hemoglobin increased with the trimester of pregnancy $\mathrm{p}<0.05$, first trimester: 4.35 (4.20 - 4.47), second trimester: $4.58(4.45-4.70)$, third trimester: 4.61 (4.14 - 5.08).

Conclusions: during pregnancy the percentage of $\mathrm{Hb} \mathrm{Alc}$ increases with the trimester of pregnancy, so it is important to determine its reference value for better metabolic control of pregnant women.

Keywords: glycosylated hemoglobin, pregnancy, non-enzymatic glycosylation, HbA1c.
Métodos: estudio observacional analítico transversal en el Hospital Ginecobstétrico «Manuel Fajardo» durante el segundo semestre del año 2016 a 100 embarazas sanas que asistieron al control prenatal; y un grupo control de 150 mujeres sanas no embarazadas mayores de 17 años en la población correspondiente al municipio Güines las que cumplieron con los criterios de inclusión y exclusión. Se realizó determinaciones de hemoglobina glucosilada y glicemia en ayunas.

Resultados: las embarazadas sanas el promedio de hemoglobina glucosilada fue menor que en las no gestante respectivamente $4.50 \%$ (4.41- 4.49) contra 5.21\% (5.165.26), $\mathrm{P}<0,001$. En el grupo de embarazada sanas la hemoglobina glucosilada aumentó con el trimestre del embarazo $\mathrm{p}<0,05$, primer trimestre: $4.35 \quad(4.20 \quad 4.47)$, segundo trimestre: 4.58 (4.45 4.70), tercer trimestre: 4.61 (4.14 5.08).

Conclusiones: durante el embarazo el porcentaje de $\mathrm{Hb} \mathrm{A} 1 \mathrm{c}$ aumenta con el trimestre del embarazo, por lo que es importante determinar su valor de referencia para un mejor control metabólico de las gestantes.

Palabras claves: hemoglobina glucosilada, embarazo, glucosilación no enzimática, HbA1c.

\section{Introducción.}

En individuos normales se encuentran postnatalmente tres tipos de hemoglobinas. La hemoglobina A, HbA (á2,â2), HbA2(á2,ä2) y la hemoglobina fetal $\operatorname{HbF}(a ́$ 2,ã2). La hemoglobina A está constituida por una parte prostética llamada hemo que es idéntico en todas las variaciones de la hemoglobina humana. La parte proteica de la molécula (globina) consta de cuatro cadenas polipeptídicas, dos cadenas alfa con 141 aminoácidos y dos cadenas betas con146 aminoácidos. 
La hemoglobina glucosilada se forma de modo no enzimático mediante una reacción de dos pasos. La primera reacción es rápida donde por condensación reversible se unen el grupo carbonilo de la glucosa y el grupo amino de la valina terminal de la cadena beta de la hemoglobina se forma una aldimina lábil o base de Schiff; a continuación, la aldimina experimenta lenta e irreversiblemente una reorganización de Amadori y se convierte en una cetoamina más estable. 1

Las hemoglobinas glucosiladas se designan como HbA1a $(<1 \%), \mathrm{HbA} 1 \mathrm{~b}(<2 \%), \mathrm{HbA1c}(3$ \%). La Federación Internacional de Química Clínica define actualmente a la $\mathrm{Hb} \mathrm{A} 1 \mathrm{c}$ como la hemoglobina A que se glucosila de forma irreversible en una o ambas valinas N-terminales de las cadenas â de la molécula tetramérica de la hemoglobina.2

La $\mathrm{Hb} \mathrm{A} 1 \mathrm{c}$ se eleva de dos a tres veces en los pacientes diabéticos. Se ha utilizado como un índice del control metabólico de la diabetes durante las 4 a 8 semanas anteriores. Como respuesta al crecimiento rápido del feto y de la placenta, así como las demandas crecientes, la mujer embarazada sufre cambios en el metabolismo de carbohidratos, lípidos y proteínas tanto numerosos como intensos. En verdad ningún otro acontecimiento fisiológico en la vida postnatal induce alteraciones metabólicas tan profundas.3, 4

La embarazada cambia con rapidez de un estado postpandrial caracterizado por un aumento sostenido del nivel de glucosa a un estado de ayunas caracterizado por descenso sanguíneo de la glucosa y algunos aminoácidos. Al mismo tiempo que son más altas las concentraciones plasmáticas de ácidos grasos libres y colesterol. Las complicaciones relacionadas con el embarazo y la diabetes pueden afectar tanto al feto como a la madre. El 2-5 \% de todas las embarazadas no diabéticas padecen diabetes gravídica, que es más frecuente entre las mujeres negras, latinas e indígenas norteamericanas. Después del embarazo, se detecta diabetes tipo 2 en el 5-10 \% de estas mujeres y el resto corre un mayor riesgo (20-50 \%) de padecer diabetes en los siguientes 5 a 10 años.5

En mujeres embarazadas y con diabetes mellitus pregestacional (tipo 1 o 2) y gestacional la determinación de la hemoglobina glucosilada tiene particular importancia pues se ha visto que su valor correlaciona con el desarrollo de malformaciones congénitas, con la ganancia de peso del producto (peso bajo al nacimiento y macrosomía) y con la presencia de complicaciones neonatales.6,7

Los cambios hematológicos producidos durante el embarazo normal que resultan en un aumento del volumen sanguíneo a expensas de un incremento tanto del plasma como de eritrocitos, siendo mayor el incremento del plasma. así como a pesar de haber un aumento de la eritropoyesis, la concentración de hemoglobina y el hematocrito descienden en grado leve y como consecuencia de las alteraciones metabólicas del embarazo, la glicación de proteínas se modifica y con ello el valor de las hemoglobinas glucosiladas. Valores diferentes de 
hemoglobina glucosilada ha sido informada, así como un comportamiento diferente en cada trimestre. 8

El propósito de este estudio es caracterizar la hemoglobina glucosilada (HbA1c) en los diferentes trimestres del embarazo. No existen antecedentes de estudios similares en la población del municipio Güines, ni se encontraron similares en Cuba en la bibliografía consultada, de ahí su importancia.

\section{Métodos}

Se realizó un estudio observacional analítico transversal en el Hospital Ginecobstétrico «Manuel Fajardo» durante el segundo semestre del año 2016 en la población femenina del municipio Güines, provincia Mayabeque.

El universo estuvo formado por un total de 370 embarazas. Para la selección de la muestra se utilizó un muestreo no probabilístico intencional, quedando constituido el grupo muestra por 100 embarazas sanas y el grupo control por 150 mujeres sanas no embarazadas, las cuales cumplieron con los criterios de inclusión y exclusión.

Criterios de inclusión para el grupo muestra:

- Embarazas sanas.

- Peso adecuado.

- Mayores de 17 años y menores de 36.

Criterios de exclusión para el grupo muestra:

- Si tiene antecedentes patológicos personales y heredofamiliares de hipertensión arterial o diabetes mellitus clínica o gestacional.

- Si tiene antecedentes ginecoobstétricos de abortos espontáneos y/o productos macrosómicos (recién nacido de más de 400 gramos).

- Sobrepeso, obesas y peso deficiente.

Criterios de inclusión para el grupo control:

- Mujeres sanas.

- Peso adecuado.

- Mayores de 17 años y menores de 36.

Criterios de exclusión para el grupo control: 
- Si tiene antecedentes patológicos personales y heredofamiliares de hipertensión arterial o diabetes mellitus clínica.

- Si tiene antecedentes ginecoobstétricos de abortos espontáneos y/o productos macrosómicos (recién nacido de más de 400 gramos).

- Sobrepeso, obesas y peso deficiente.

Se empleó un método inmunoturbidimétrico utilizando el kit de reactivos para la determinación cuantitativa de la hemoglobina A1c en sangre humana.

El método se basa en la interacción antígeno- anticuerpo para la determinación directa de la hemoglobina glucosilada (HbA1c) en sangre total anticoagulada con ácido etilendiaminotetraacético (comúnmente denominado EDTA). La hemoglobina total y la HbA1c tienen la misma capacidad de ligarse específicamente a partículas de látex. Cuando se añaden anticuerpos monoclonales de ratón anti HbA1c humana (R2), se forma un complejo látexHbA1c-anticuerpo monoclonal de ratón anti HbA1c humana. La reacción de anticuerpos policlonales de cabra con los anticuerpos monoclonales de ratón produce una aglutinación que es proporcional a la cantidad de $\mathrm{HbA1c}$ absorbida sobre la superficie de las partículas de látex. La aglutinación es medida como absorbancia y el valor de HbA1c se obtiene por una curva de calibración en un autoanalizador químico INLAB, en el laboratorio de bioquímica clínica del Hospital Clínico Quirúrgico «Hermanos Ameijeiras».9

El método presenta un coeficiente de variación (CV) en la serie menor de un $2 \%$ y entre las series menor de un $2,8 \%$ y un valor de referencia (VR) no diabéticos $<6 \%$.

Los datos fueron introducidos en una base de datos en el programa Excel para Windows en una PC Pentium IV, para su posterior análisis estadístico en el sistema Infostat versión 9, en el que se determinó el porciento de hemoglobina glucosilada (HbA1c) en mujeres sanas embarazadas. Los resultados fueron mostrados en tablas y gráficos.

Se informan las medias, la desviación típica y el intervalo de confianza de un $95 \%$ de cada una de las variables estudiadas. Se aplicó ANOVA de una vía, con prueba Post- hoc de Tukey para encontrar las diferencias entre grupos. Las interrelaciones entre variables se analizaron con el coeficiente de correlación de Pearson. En ambos se consideró significativa la asociación cuando la p asociada fue menor de 0.05 .

\section{Resultados}

Se muestra la media de las variables, edad, peso, talla, índice de masa corporal (IMC), paridad de las mujeres sanas embarazadas y no embarazadas (tabla 1). 
Tabla 1. Descripción de las variables seleccionadas de la población de estudio

\begin{tabular}{c|c|c} 
Variable & Embarazadas & No embarazadas \\
\hline Edad cronológica & $24.5( \pm 5.3)$ & $23.0( \pm 3.9)$ \\
\hline Peso $($ Kg) & $56.3( \pm 6.8)$ & $57.6( \pm 6.3)$ \\
\hline Talla $(\mathbf{c m})$ & $160( \pm 0.1)$ & $160( \pm 0.1)$ \\
\hline IMC $\left(\mathbf{K g} / \mathbf{m}^{2}\right)$ & $22.2( \pm 2.0)$ & $21.7( \pm 1.5)$ \\
\hline Paridad & $1.0( \pm 0.7)$ & $1.0( \pm 0.5)$
\end{tabular}

Fuente: Elaboración propia.

El valor porcentual de la HbA1c que fue significativamente menor en las embarazas sanas (E) durante toda la gestación $(4.5$ vs $5.25 \%$, p<0.001) que en las no embarazadas $(\mathrm{NE})$ (gráfico 1).

Gráfico 1. Valor porcentual de la HbA1c en los grupos estudiados

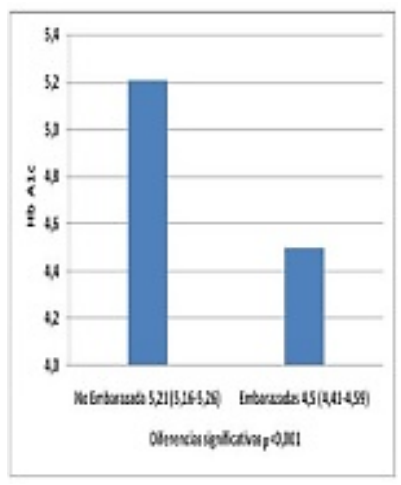

Fuente: Elaboración propia.

Además, en el grupo E, la HbA1c aumentó con el trimestre del embarazo (4.35; 4.57; 4.61, $\mathrm{p}<0.05$ ) respectivamente (gráfico 2).

Gráfico 2. Valor de $\mathrm{Hb}$ A1c en los trimestres del embarazo.

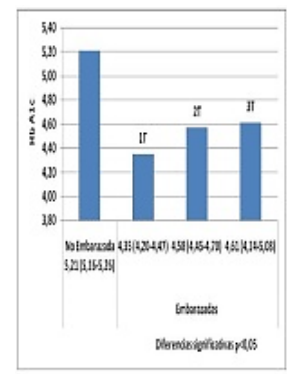

Fuente: Elaboración propia. 


\section{ADDigigital}

ISSN: 2697-3391

Vol. 1, N¹, p. 39-49, abril-junio, 2018

Aumentó la HbA1c con el trimestre del embarazo en las embarazadas de 26-30 años y de 31-35 años (tabla 2).

Tabla 2. Valor porcentual de HbA1c de acuerdo con la edad cronológica y el trimestre del embarazo

\begin{tabular}{c|c|c|c|c} 
Grupo & 1T & 2T & 3T & Significación \\
\hline I (18-25 años) & $4.31 \pm 0.40$ & $4.62 \pm 0.46$ & $4.56 \pm 0.48$ & $\mathrm{p}>0.05$ \\
\hline II (26-30 años) & $4.39 \pm 0.31$ & $4.44 \pm 0.39$ & $4.93 \pm 0.15$ & $\mathrm{p}>0.05$ \\
\hline III (31-35 años) & $4.33 \pm 0.54$ & $4.54 \pm 0.37$ & $4.55 \pm 0.51$ & $\mathrm{p}>0.05$
\end{tabular}

Fuente: Elaboración propia.

Se observó una relación lineal entre la glicemia y la HbA1c en las mujeres sanas no embarazada y embarazada (gráfico 3).

Gráfico 3. Correlación entre la HbA1c y la glicemia de la población estudiada.

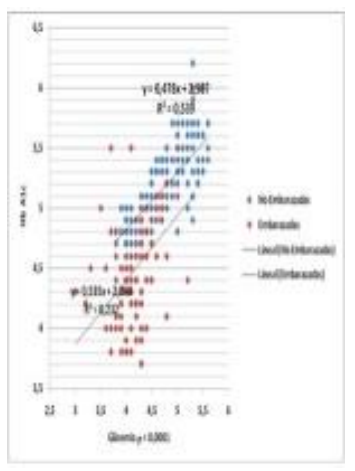

Fuente: Elaboración propia.

\section{Discusión}

En las mujeres embarazadas sanas el valor porcentual de la HbA1c es significativamente menor al de las mujeres no embarazadas lo que coincide con otros estudios realizados. ${ }^{10,11}$

El valor porcentual bajo de HbA1c durante el embarazo se atribuye a diferentes factores, entre ellos destacan las alteraciones en el metabolismo de los carbohidratos que disminuyen la concentración sérica de la glicemia en el ayuno, la concentración de la hemoglobina que desciende en grado leve y el aumento del volumen plasmático de la madre que comienza a aumentar durante el primer trimestre, se expande con mayor rapidez en el segundo trimestre y en el tercero se incrementa de forma mucho más lenta hasta alcanzar una meseta en el tercer trimestre. $^{6}$ 
Un estudio realizado en Japón ${ }^{12}$ muestra una disminución significativa de la HbA1c en el segundo trimestre, resultado que difiere con la presente investigación donde aumenta durante los tres trimestres. Otros autores plantean una disminución significativa de la $\mathrm{HbA} 1 \mathrm{c}$ en el primer y segundo trimestre en comparación con el tercero. ${ }^{13,14}$

No se encuentra una relación significativa con la edad cronológica en esta investigación. Los efectos de la edad sobre la HbA1c son controvertidos, ya que algunos estudios ${ }^{15}$ muestran un incremento de aproximadamente un $0,1 \%$ por década a partir de los 30 años en personas sanas, situación que podría explicarse por el aumento de la glucemia en ayunas y la disminución de la tolerancia a la glucosa que se observa con la edad, o también pudiera ser debida a la disminución en el recambio de eritrocitos, como resultado de la reducción de la tasa metabólica asociada con el envejecimiento. Sin embargo, otros estudios ${ }^{16}$ con selección de controles sanos, mediante pruebas de tolerancia a la glucosa oral, no han demostrado que la edad afecte los niveles de HbA1c.

Existe una relación lineal entre la glicemia y la HbA1c en las mujeres sanas no embarazadas y embarazadas, resultados iguales reportan otros estudios. ${ }^{17,18}$

El presente estudio tiene la importancia de ser el primero que se realiza en la población del municipio Güines, en mujeres sanas embarazadas y no embarazadas y las cifras de porcentaje de HbA1c durante el embarazo y en cada uno de los trimestres del mismo, servirán de referencia para evaluar el grado de control metabólico de las mujeres sanas embarazadas y con diabetes mellitus pregestacional (tipo 1 y 2) y gestacional.

Se concluye que durante el embarazo el porcentaje de $\mathrm{Hb}$ A1c aumenta con el trimestre del embarazo, por lo que es importante determinar su valor de referencia para un mejor control metabólico de las embarazadas.

\section{Conflictos de intereses}

Los autores declaran que no existen conflictos de intereses para la publicación del artículo.

\section{Referencias bibliográficas.}

Guerra M, Torres AL, Alvarado M, Bustamante T, Lavalle C, Luján D. Relación de los niveles de HbA1c (\%) y de fructosamina (mg/dl) en sujetos saludables y diabéticos tipo 1.v. UniversitasScientiarum Revista de la Facultad de Ciencias. 2007; 12(1):5565.

Vargas E.A, Gómez JH, Conde JM. Medición de la hemoglobina glucosilada capilar como tamizaje en diabetes mellitus tipo 2. MedIntMéx [Internet]. 2014[citado10 Oct 2015]; 
30: 538-45. Disponible en: http://www.medigraphic.com/pdfs/medintmex/mim2014/mim145f.pdf

Cruz J, Hernández P, Grandía R, Lang J, Isla A, González K, et al. Consideraciones acerca de la diabetes mellitus durante el embarazo. Rev Cubana Endocrinol [Internet]. 2015 Abr [citado 3 Abr 2016]; 26(1): 47-65. Disponible en: http://scielo.sld.cu/scielo.php?script=sci_arttext\&pid=S1561$29532015000100005 \& \operatorname{lng}=\mathrm{es}$

Santiesteban S, Oliva Rodríguez J. Semiología obstétrica. En: Rigol Ricardo O. Obstetricia y Ginecología. La Habana: Ciencias Médicas; 2010. p. 5572.

Capítulo 5 Fisiología materna. En: Cunningham Gary F. Williams Obstetricia. 23 ed. México: Mc GrawHill Interamericana; 2011. p. 10735.

Shobha P, Mathen S, Abraham J. Glycosylatedhemoglobinvalues in nondiabeticpregnantwomen in thethirdtrimester and adverse fetal outcomes: anobservationalstudy. J FamilyMed Prim Care [Internet]. 2016Jul-Sep;5(3):646-51. Disponible en: https://www.ncbi.nlm.nih.gov/pmc/articles/PMC5290776/

Ye M, Liu Y, Cao X, Yao F, Liu B, Li Y, et al. Theutility of HbA1c forscreeninggestational diabetes mellitus and itsrelationshipwith adverse pregnancyoutcomes. Diabetes Res ClinPract [Internet]. 2016Apr; 114:43-9 [citado 9 Oct 2016]. Disponible en: https://www.ncbi.nlm.nih.gov/pubmed/27103368

Adaptaciones maternas del embarazo. En: Cunninghan GF, Gant NF, Leveno KJ, Gilstrap LC, Hauth JC, Wenstrom KD. Williams Obstetricia. 21 ed. La Habana: Editorial Ciencias Médicas; 2007. p. 141-69.

Piermattei C. Hemoglobina HbA1c directa para la determinación cuantitativa de la hemoglobina A1c en sangre humana. Roma: CPM científica, 2012.

Mosca A, Paleari R, Dalfrà MG, Di Cianni G, Cuccuru I, Pellegrini G, et al. Reference intervalsforhemoglobin A1c in pregnantwomen: data fromnaltalianmulticenterstudy. ClinChem [Internet]. 2006 Jun[citado 15 Feb 2017];52(6):1138-43. Disponible en: http://www.ncbi.nlm.nih.gov/pubmed/16601066

Connor CO, O'Shea PM, Owens LA, Carmody L, Avalos G, Nestor L. Trimesterspecificreferenceintervalsforhaemoglobin A 1c (HbA 1c) in pregnancy. ClinChem [Internet]. 2012[citado 15 Feb 2017];50 (5):9059. Disponible en: http://atlanticdipireland.com/wp-content/uploads/2016/01/trimester-specificreference-intervals-for-glycated-haemoglobin-in-pregnancy.pdf 
Hiramatsu Y, Shimizu I, Omori Y, Nakabayashi M. Determination of referenceintervals of glycatedalbumin and hemoglobin A1c in healthypregnantJapanesewomen and analysis of their time courses and influencingfactorsduringpregnancy. Endocr $\mathrm{J}$ [Internet]. 2012 Dec [citado 15 Feb 2017];59(2):145-51. Disponible en: https://www.ncbi.nlm.nih.gov/pubmed/22166921

Lowe LP, Metzger BE, Dyer AR, Lowe J, McCance DR, Lappin TR, et al. Hyperglycemia and Adverse PregnancyOutcome (HAPO) Study: associations of maternal A1C and glucosewithpregnancyoutcomes. Diabetes Care [Internet]. 2012[citado 15 Feb 2017]; 35(3):574-80. Disponible en: http://europepmc.org/abstract/med/22301123

Nathan DM, Kuenen J, Borg R, Zheng H, Schoenfeld D, Heine RJ. Translatingthe A1C assayintoestimatedaverageglucosevalues. Diabetes Care [Internet]. 2008 Aug[citado 30 Oct 2017];31 (8):1473-78. Disponible en: http://care.diabetesjournals.org/content/31/8/1473.short

Sharma B, Mahajan P, Prasad S, Pandey R, Singh J, Sodhi KS. Glycatedhaemoglobinlevels in no diabetics: theeffecttoageing. medicalscience [Internet]. 2013 Jul-Dec[citado 13 Oct 2017];2 (2): 116-24. Disponible en: https://www.ejmanager.com/mnstemps/69/69-1383930664.pdf?t=1556653636

Goyal G, PanagK, Sheenam S, Singh J. High glycatedhemoglobinlevels in corelationwithageing in non-diabetics. Int J Med Res Prof [Internet]. 2015 [citado 30 Oct 2016];1(3): 35-39. Disponible en: http://admin.ijmrp.com/Upload/IJMRP\%201(3)\%2035-39.pdf

Amador Rodríguez L, Sosa Pérez JC, BuchacaFexas EF, Fernández Valdés F, Bermudéz Rojas SA, Mora I. Niveles de hemoglobina glucosilada y su correlación con las glucemias de ayuno y postprandial en un grupo de pacientes diabéticos. Acta Médica [Internet]. 2015[citado 23 Oct 2015];16(1). Disponible en: http://bvs.sld.cu/revistas/act/vol16_1_15/act04115.htm

Orellana R. La hemoglobina glucosiladas. RevInvSci [Internet]. 2014[citado 21 Mar 2018]; 3(1):3-9. http://www.revistasbolivianas.org.bo/scielo.php?pid=S231302292014000100001\&script=sci_arttext

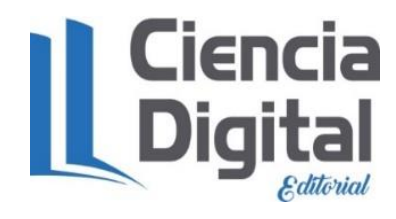




\section{PARA CITAR EL ARTÍCULO INDEXADO.}

Arencibia Fundora, Y., Piloto Rivera, I., Gonzáles Paneque, A., \& Bello Ferro, M. (2018). Caracterización de la hemoglobina glucosilada A1c en embarazas sanas. Anatomía Digital, 1(1), 39-49. https://doi.org/10.33262/anatomiadigital.v1i1.1040

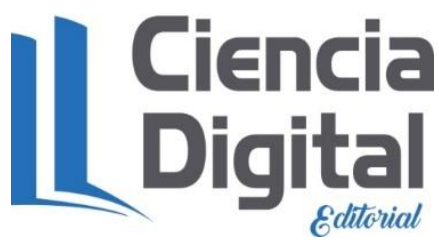

El artículo que se publica es de exclusiva responsabilidad de los autores y no necesariamente reflejan el pensamiento de la Revista Anatomía Digital.

El artículo queda en propiedad de la revista y, por tanto, su publicación parcial y/o total en otro medio tiene que ser autorizado por el director de la Revista Anatomía Digital.
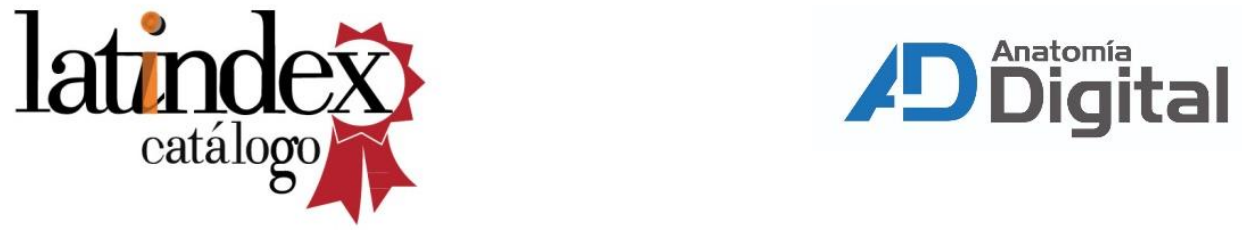